\title{
Multiple Biomarker Panels for Early Detection of Breast Cancer in Peripheral Blood
}

\author{
Fan Zhang, ${ }^{1,2}$ Youping Deng, ${ }^{3}$ and Renee Drabier ${ }^{1}$ \\ ${ }^{1}$ Department of Academic and Institutional Resources and Technology, University of North Texas Health Science Center, \\ Fort Worth, 76107, USA \\ ${ }^{2}$ Department of Forensic and Investigative Genetics, University of North Texas Health Science Center, \\ Fort Worth, 76107, USA \\ ${ }^{3}$ Department of Internal Medicine Kidston House, Rush University Medical Center, 630 S. Hermitage Avenue, Room 408, \\ Chicago, IL 60612, USA
}

Correspondence should be addressed to Renee Drabier; renee.drabier@unthsc.edu

Received 12 September 2013; Accepted 8 November 2013

Academic Editor: Zhongming Zhao

Copyright (C) 2013 Fan Zhang et al. This is an open access article distributed under the Creative Commons Attribution License, which permits unrestricted use, distribution, and reproduction in any medium, provided the original work is properly cited.

\begin{abstract}
Detecting breast cancer at early stages can be challenging. Traditional mammography and tissue microarray that have been studied for early breast cancer detection and prediction have many drawbacks. Therefore, there is a need for more reliable diagnostic tools for early detection of breast cancer due to a number of factors and challenges. In the paper, we presented a five-marker panel approach based on SVM for early detection of breast cancer in peripheral blood and show how to use SVM to model the classification and prediction problem of early detection of breast cancer in peripheral blood. We found that the five-marker panel can improve the prediction performance (area under curve) in the testing data set from 0.5826 to 0.7879 . Further pathway analysis showed that the top four five-marker panels are associated with signaling, steroid hormones, metabolism, immune system, and hemostasis, which are consistent with previous findings. Our prediction model can serve as a general model for multibiomarker panel discovery in early detection of other cancers.
\end{abstract}

\section{Introduction}

Traditional methods mostly used for early detection have been regular and periodic self-examination and annual or biannual checkups using mammography and analysis of tissue biopsies. But mammography as a screening tool for early detection has many drawbacks. For example, mammography may not detect small tumors and is often unsatisfactory for younger women, who typically have dense breast tissue. And if a patient does have a suspicious mammogram, a biopsy will probably be done to make the diagnosis. Obtaining tissue biopsies can be difficult for several reasons, including small size of lump, lack of available medical facilities, and patients' reluctance to undergo invasive procedures due to scaring and costs.

In recent years, functional genomics studies using DNA microarrays have been shown effective in differentiating between breast cancer tissues and normal tissues by measuring thousands of differentially expressed genes simultaneously [1-3]. However, early cancer detection and treatment are still challenging. One reason is that obtaining tissue samples for microarray analysis can be still difficult. Another reason is that the signatures of gene expression difference between normal and cancer obtained in different studies are not sufficiently reproducible or informative to be prognostically useful, although they do give valuable insights into the pathogenesis and biology of human tumor metastasis [4]. Moreover, the fact that breast cancer is not a single homogeneous disease but consists of multiple disease status, each arising from a distinct molecular mechanism and having a distinct clinical progression path $[5,6]$, makes the disease difficult to detect in early stages.

To address these issues, a novel and minimally invasive test that uses easily obtained peripheral blood for breast 
cancer detection has been developed [7, 8]. For example, Sharma et al. used microarrays and nearest-shrunkencentroid method to analyze the expression pattern of 1,368 genes in peripheral blood cells of 24 women with breast cancer and 32 women with no sign of this disease. The study found that a blood-based gene expression test can be developed to detect breast cancer early in asymptomatic patients [8]. Aarøe et al. collected peripheral blood from 67 breast cancer samples and 63 normal samples, identified a set of 738 differentially expressed probes, and achieved an estimated prediction accuracy of $79.5 \%$ with a sensitivity of $80.6 \%$ and a specificity of $78.3 \%$ [7].

There is a need for more reliable diagnostic tools for early detection of breast cancer in peripheral blood which can achieve high prediction accuracy with as few genes as possible and to reduce the required examination of a large number of genes which increases the dimensionality, computational complexity, and clinical cost of diagnosis [8]. Li estimated that five or six genes rather than 37 or 738 would be sufficient for the early detection of breast cancer, based on colon cancer, leukemia, and breast cancer [8]. Therefore, it is desirable to adopt a "multimarker panel" concept and nontrivial computational methods that can integrate microarray measurement of multiple differential gene expression levels between disease and controls to achieve good performance for clinical genomic development of biomarkers [9].

Support vector machine (SVM) has several unique characteristics as a research tool for prediction in cancer classification applications. One unique characteristic as a specific type of learning algorithm is that it is characterized by the capacity control of the decision function, the use of the kernel functions, and the sparsity of the solution [10]. The second unique characteristic of SVM is that it is established on the unique theory of the structural risk minimization principle to estimate a function by minimizing an upper bound of the generalization error and therefore very resistant to the overfitting problem, eventually achieving a high generalization performance. The third unique characteristic of SVM is that training SVM is equivalent to solving a linearly constrained quadratic programming problem so that the solution of SVM is always unique and globally optimal, unlike neural networks training, which requires nonlinear optimization with the danger of getting stuck at local minima.

For classification and prediction of breast cancer samples, these unique characteristics make SVM appealing as compared with regression-based models or neural network as seen in [11-13]. For example, Liu et al. used SVM to predict the state of breast cancer and found that SVM outperformed $\mathrm{K}$-means cluster and artificial neural network [11]. Henneges et al. applied oscillating search algorithm for feature selection (OSAF) to iteratively improve features for training of Support vector machines (SVM) to better predict breast cancer [12]. They selected 35 out of 51 nucleosides/ribosylated metabolites in the urine of breast cancer women and controls by LCITMS coupling for subsequent computational analyses, and they identified 44 pairwise ratios of metabolite features by iterative optimization of SVM. Liu et al. combined genetic algorithm (GA) and all paired (AP) support vector machine
TABLE 1: Statistics of samples.

\begin{tabular}{lccc}
\hline & \#health & \#cancer & \#total \\
\hline Training group & 32 & 34 & 66 \\
Testing group & 31 & 33 & 64 \\
\hline Total & 63 & 67 & 130 \\
\hline
\end{tabular}

(SVM) methods to determine the predictive features for multiclass breast cancer categorization [13].

There has not been any report until this study that applied SVM to the development of multimarker panels for early detection of breast cancer based on peripheral blood. Based on a neural network approach to multibiomarker panel development for LC/MS/MS proteomics profiles we developed [14], we propose for the first time a multimarker panel development solution for early detection of breast cancer in peripheral blood by using a SVM and show how to use SVM to model the classification and prediction problem of early detection of breast cancer in peripheral blood.

\section{Methods and Materials}

2.1. Peripheral Blood Data Collection. The peripheral blood data are publicly available through the GEO database with the accession number GSE16443 [7] and were collected with the purpose of determining the potential of gene expression profiling of peripheral blood cells for early detection of blood cancer. It consists of 130 samples with 67 cases and 63 controls. We downloaded the 130 samples which contain 32,879 probes. Then we randomly divided the 130 samples into two groups: group $\mathrm{A}$ as a training group and group B as a testing group (Table 1 ).

2.2. Normalization. Per sample normalization was performed to normalize for staining intensity variations among samples. All expression data on a sample were normalized to the 50th percentile of $\log$ base 2 of all values on that sample. First, $\log$ ratio base 2 transformation was used to transform the data. And then for each probe the median of the log summarized values from all the samples was calculated and subtracted from each of the samples.

2.3. Linear Mixed Model. We used the ABI Human Genome Survey Microarray Version 2 to manage and map probe IDs. A full factorial model was used to represent the fixed effect and the random effect which are used to account for group and probe. The expression log ratios value is the final quantity that is fit by a separate analysis of the variance (ANOVA) statistical model for each probe as $y_{i j}$ using the following:

$$
y_{i j}=\mu+T_{i}+S_{j}+\varepsilon_{i j}
$$

where $S_{j} \in N\left(0, \sigma_{1}^{2}\right), \varepsilon_{i j} \in N\left(0, \sigma^{2}\right)$. Here, $\mu$ is the mean expression value, $T_{i}$ is the fixed group effect (caused by the experimental conditions or treatments being evaluated), $S_{j}$ is the random sample effect (random effects from either individual biological samples or sample preparations), and $\varepsilon_{i j}$ is the within-groups errors. All random effects are assumed 
independent of each other and independent of the withingroups errors $\varepsilon_{i j}$.

2.4. Statistics. Statistical significance was measured by a three-step method. First, we conducted the above linear mixed model to obtain the $P$ value of the significance for the group effect. Then we calculated the FDR adjusted $P$ value. Last, we calculated the FDR $q$ value using the StoreyTibshirani method [15]. We chose a significance screening filter $(q<0.01)$ to select genes of which we estimated significant differences in the health and breast cancer samples.

2.5. Support Vector Machine Analysis. The classification problem of breast cancer can be restricted to consideration of the two-class problem without loss of generality (breast cancer and normal). We used a support vector machine- (SVM-) based methods [16] to develop the classifier for breast cancer from peripheral blood. And then we applied the classifier to predict blind dataset of breast cancer from peripheral blood.

For the use of the support vector machine as an appropriate tool for prediction of the breast cancer, a three-way data split is applied for training, validation, and testing. The training set is used for learning to fit the parameters of the classifier. The validation set is used to tune the parameters of the classifier. And the testing set is used only to assess the performance of the fully-trained classifier. We first randomly split the data into two groups: group A (training group) and group B (testing group), with roughly equal size. Then we use the $k$-fold cross validation on the training group to find the "optimal" parameters for the classifier. Group A is randomly partitioned into $k$ subsamples. For each subsample, a cross section of the data is flagged for use as the validation set, and a new model is created by training on the remaining data which are the training set and not in the subsample. The cross validation process is then repeated $k$ times (the folds), with each of the $k$ subsamples used exactly once as the validation data. The $k$ results from the folds then can be averaged to produce a single estimation. The testing group is used as testing set.

We chose each combination of $N(N=5$ for fivemarker panel) out of all the 42 genes differentially expressed in the training group as inputs to the SVM. In order to find the optimal classifier, we presented an optimization method that measures the area under the curve (AUC) for receiver operating characteristics (ROC). In this scheme, we first train SVM for each combination in the training set with 5-fold cross validation. Then, we measured the AUC for each combination in the validation set. Lastly, the optimal combination $C^{*}$ was determined by

$$
C^{*}=\underset{C}{\operatorname{argmax}} \operatorname{AUC}\left(\operatorname{SVM}_{C}, V\right)
$$

where AUC is the area under the ROC curve of SVM prediction, SVM is the support vector machine, $C$ is combination of picking five out of the 42 genes, and $V$ is the validation set of training group.

Fivefold cross validation was used to increase the number of estimates and improve the accuracy of the prediction model by avoiding the overfitting. In 5 -fold cross validation, the original sample is randomly partitioned into 5 subsamples. Of the 5 subsamples, a single subsample is retained as the validation data for testing the model, and the remaining 4 subsamples are used as training data. The cross validation process is then repeated 5 times, with each of the 5 subsamples used exactly once as the validation data. The 5 results from the folds then can be averaged to produce a single estimation. The advantage of this method over repeated random subsampling is that all observations are used for both training and validation, and each observation is used for validation only once.

2.6. Pathway Analysis. The Integrated Pathway Analysis Database (IPAD) (http://bioinfo.hsc.unt.edu/ipad/) [17] is used for pathway analysis.

\section{Results}

We downloaded from the Gene Expression Omnibus (accession number GSE16443) [7] the 130 samples with 67 breast cancer and 63 healthy women. After we randomly divided the 130 samples into two groups, group A as training group and group B as testing group (Table 1), we obtained 32 healthy samples and 34 cancer samples in the training set and validation set and 31 healthy samples and 33 cancer samples in the testing set.

We obtained 42 markers in the training group with $q$ value $<0.01$. No data from the testing set were utilized in (1) identification of peripheral blood markers or (2) development of the SVM model.

An SVM model with 5-fold cross validation was built on all 42 markers in the training group. We obtained a high performance $(\mathrm{AUC}=1.0$, precision $=94.4 \%$, accuracy $=$ $97.0 \%$, sensitivity $=100.0 \%$, and specificity $=93.8 \%)$ for the training group but a low performance (AUC $=0.58$, precision $=58.3 \%$, accuracy $=57.8 \%$, sensitivity $=63.6 \%$, and specificity $=51.6 \%$ ) for the testing group (Figure 1 ). The result shows that using all markers as a predictor can improve the prediction accuracy only for training group but not for the testing group. Therefore, we constructed an SVM with 5fold cross validation for each combination of five out of 42 markers and trained with breast cancer cells in peripheral blood derived from 34 women diagnosed with breast cancer and 32 control women in the training group. The three-way data split was applied for training, validation, and testing. The optimal combinations were obtained by our optimization model based on the training set and validation set in the training group.

Training of the SVM was performed using radius basis function (RBF) kernels function and five-fold cross validation. Receiver operating characteristic (ROC) curve and area under curve (AUC) were calculated to help evaluating the predictive performance of the SVM. We choose $N=5$ for five-marker panel because (1) our pilot study shows that five markers can be enough to achieve a satisfied performance for prediction and classification of cancer [14], (2) previous papers from other labs estimated that five or six genes would be sufficient for the early detection of breast cancer [18], and 


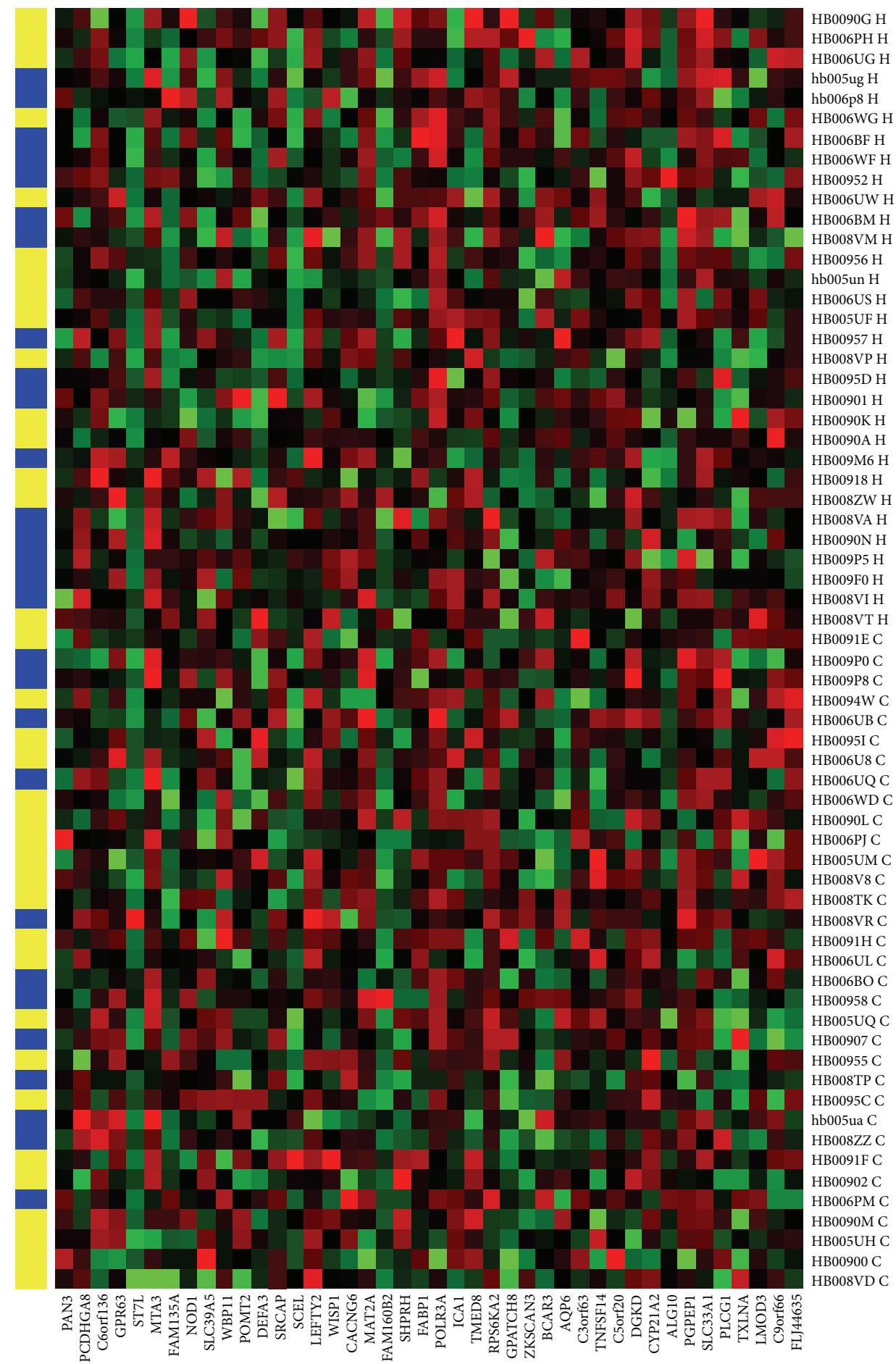

FIGURE 1: 42 biomarkers predicting the healthy and breast cancer samples in testing set. $X$-axis is the 42 biomarkers. $Y$-axis shows the 33 breast cancer and 31 healthy samples (H: healthy, blue; C: cancer, yellow).

(3) we expect to achieve high prediction accuracy for breast cancer with as few genes/proteins as possible.

In order to validate our prediction method, we compared the ROCs for the best four 5-marker panel predictions determined by our method with the ROCs for four randomly chosen 5-marker panels from 42 candidate biomarkers
(Figure 2). As shown in the Figure 2, the top four best predictions determined by our method (solid lines) have better sensitivity-specificity-tradeoff performance than those chosen randomly from 42 candidate biomarkers.

In Table 2, we show the best four five-marker panels identified, using the SVM. Two genes, BCAR3 and LEFTY2, 


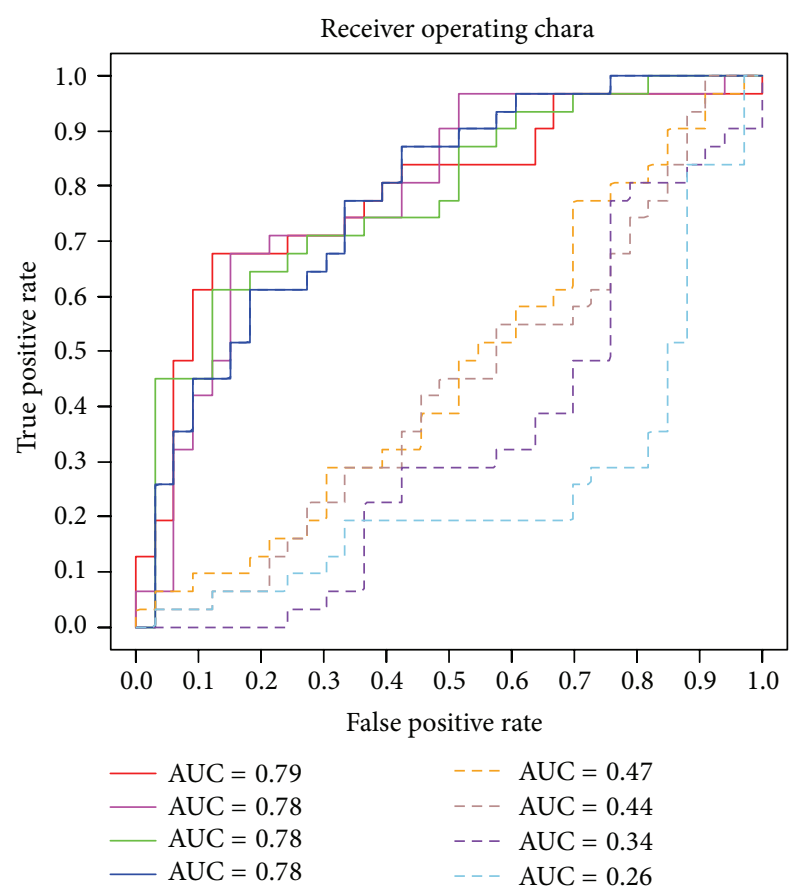

Figure 2: A comparison of best four 5-marker panel ROCs (solid lines) and randomly chosen four (out of 42 candidates) 5-marker ROCs (dotted lines).

TABLE 2: Best four five-marker panels identified.

\begin{tabular}{lcc}
\hline Panel & $\begin{array}{c}\text { Training group } \\
\text { AUC }\end{array}$ & $\begin{array}{c}\text { Testing group } \\
\text { AUC }\end{array}$ \\
\hline $\begin{array}{l}\text { PCDHGA8; LEFTY2; CACNG6; } \\
\text { BCAR3; CYP21A2 }\end{array}$ & 0.9053 & 0.7879 \\
$\begin{array}{l}\text { PCDHGA8; DEFA3; SCEL; } \\
\text { LEFTY2; BCAR3 }\end{array}$ & 0.9127 & 0.7830 \\
$\begin{array}{l}\text { DEFA3; SCEL; LEFTY2; } \\
\text { CACNG6; BCAR3 }\end{array}$ & 0.9154 & 0.7801 \\
$\begin{array}{l}\text { DEFA3; LEFTY2; CACNG6; } \\
\text { BCAR3; DGKD }\end{array}$ & 0.8897 & 0.7801 \\
\hline
\end{tabular}

are in common between the best four five-marker panels. Two genes, CACNG6 and DEFA3, are shown three times, and two genes, PCDHGA8 and SCEL, are shown twice.

Pathway analysis shows that the pathways linked with the best four five-marker panels are signaling, steroid hormones, metabolism, immune system, and hemostasis (Table 3), which are consistent with previous findings [7].

The confusion matrix and common performance metrics for both the training group and testing group for the best fivemarker panel are shown in the Table 4. Although the final accuracy is $68.75 \%$, it can be considered as an improvement if compared to the original accuracy $58.81 \%$. In addition, the AUC, a comprehensive measurement of sensitivity and specificity, is improved markedly from 0.5826 to 0.7879 (Figure 2 and Table 4).

We further evaluated our multimarker panel prediction performance by comparing our results with prediction performance in previously published findings. Sharma et al.
TABLE 3: Pathway analysis for the best four five-marker panels.

\begin{tabular}{|c|c|c|}
\hline Pathway ID & Pathway name & Molecule \\
\hline 200071 & Regulation of CDC42 activity & BCAR3 \\
\hline hsa04260 & Cardiac muscle contraction & CACNG6 \\
\hline hsa05412 & $\begin{array}{l}\text { Arrhythmogenic right } \\
\text { ventricular cardiomyopathy } \\
\text { (ARVC) }\end{array}$ & CACNG6 \\
\hline hsa05410 & $\begin{array}{l}\text { Hypertrophic cardiomyopathy } \\
\text { (HCM) }\end{array}$ & CACNG6 \\
\hline hsa05414 & Dilated cardiomyopathy & CACNG6 \\
\hline hsa04010 & MAPK signaling pathway & CACNG6 \\
\hline 194002 & Glucocorticoid biosynthesis & CYP21A2 \\
\hline 193993 & Mineralocorticoid biosynthesis & CYP21A2 \\
\hline 211976 & Endogenous sterols & CYP21A2 \\
\hline 209943 & Steroid hormones & CYP21A2 \\
\hline 196071 & $\begin{array}{l}\text { Metabolism of steroid hormones } \\
\text { and vitamins A and D }\end{array}$ & CYP21A2 \\
\hline 211897 & $\begin{array}{l}\text { Cytochrome } \mathrm{P} 450 \text {, arranged by } \\
\text { substrate type }\end{array}$ & CYP21A2 \\
\hline 211945 & $\begin{array}{l}\text { Phase 1, functionalization of } \\
\text { compounds }\end{array}$ & CYP21A2 \\
\hline 211859 & Biological oxidations & CYP21A2 \\
\hline hsa00140 & Steroid hormone biosynthesis & CYP21A2 \\
\hline 556833 & $\begin{array}{l}\text { Metabolism of lipids and } \\
\text { lipoproteins }\end{array}$ & CYP21A2 \\
\hline 1430728 & Metabolism & CYP21A2 \\
\hline 1462054 & Alpha-defensins & DEFA3 \\
\hline 1461973 & Defensins & DEFA3 \\
\hline hsa05202 & $\begin{array}{l}\text { Transcriptional misregulation in } \\
\text { cancer }\end{array}$ & DEFA3 \\
\hline 168249 & Innate immune system & DEFA3 \\
\hline 168256 & Immune system & DEFA3 \\
\hline 114508 & Effects of PIP2 hydrolysis & DGKD \\
\hline hsa00561 & Glycerolipid metabolism & DGKD \\
\hline hsa04070 & $\begin{array}{l}\text { Phosphatidylinositol signaling } \\
\text { system }\end{array}$ & DGKD \\
\hline hsa00564 & Glycerophospholipid metabolism & DGKD \\
\hline 416476 & G alpha (q) signalling events & DGKD \\
\hline 388396 & GPCR downstream signaling & DGKD \\
\hline 372790 & Signaling by GPCR & DGKD \\
\hline 162582 & Signal transduction & DGKD \\
\hline 76002 & $\begin{array}{l}\text { Platelet activation, signaling, and } \\
\text { aggregation }\end{array}$ & DGKD; LEFTY2 \\
\hline 109582 & Hemostasis & DGKD; LEFTY2 \\
\hline 1433617 & $\begin{array}{l}\text { Regulation of signaling by } \\
\text { NODAL }\end{array}$ & LEFTY2 \\
\hline 1181150 & Signaling by NODAL & LEFTY2 \\
\hline 114608 & Platelet degranulation & LEFTY2 \\
\hline 76005 & $\begin{array}{l}\text { Response to elevated platelet } \\
\text { cytosolic Ca2+ }\end{array}$ & LEFTY2 \\
\hline hsa04350 & TGF-beta signaling pathway & LEFTY2 \\
\hline 1266738 & Developmental biology & LEFTY2 \\
\hline
\end{tabular}

identified a panel of 37 genes that permitted early detection with the classification accuracy of $82 \%$ [8], and Aarøe et al. identified a set of 738 differentially expressed probes that achieved an estimated prediction accuracy of $79.5 \%$ with a 
TABle 4: Prediction result for the best 5-marker panel.

\begin{tabular}{lcccc}
\hline \multirow{2}{*}{ Predicted } & \multicolumn{2}{c}{ Training group } & \multicolumn{2}{c}{ Testing group } \\
& Cancer & Normal & Cancer & Normal \\
\hline Cancer & 29 & 6 & 21 & 8 \\
Normal & 5 & 26 & 12 & 23 \\
Precision & & $82.86 \%$ & & $72.41 \%$ \\
Accuracy & & $83.33 \%$ & & $68.75 \%$ \\
Sensitivity & & $85.29 \%$ & & $63.64 \%$ \\
Specificity & & $81.25 \%$ & & $74.19 \%$ \\
\hline
\end{tabular}

sensitivity of $80.6 \%$ and a specificity of $78.3 \%$ [7]. Considering that their methods were not applied to independent testing group randomly separated from training group but used $k$ fold cross validation where the original sample was randomly partitioned into $k$ subsamples and of the $k$ subsamples, a single subsample was retained as the validation data for testing the model, and the remaining $k-1$ subsamples were used as training data, our prediction performance actually outperformed them. When we applied an SVM and 5-fold cross validation with our best 5 -marker panel to the training group of 34 women with breast cancer and 32 healthy women controls, we obtained a higher performance than these previously published findings (precision $=82.86 \%$, accuracy $=83.33 \%$, sensitivity $=85.29 \%$, and specificity $=81.25 \%$, Table 4 ). We believe that our approach is a significant success, considering that we only used five gene markers in a panel to achieve the prediction performance $(\mathrm{AUC}=0.7879$, precision $=72.41 \%$, accuracy $=68.75 \%$, sensitivity $=63.64 \%$, and specificity $=$ $74.19 \%)$.

\section{Discussions}

In this study, we incorporate the use of a three-way data split in combination with an enumeration method based on SVM. It is a reasonably straightforward application of existing methods and achieves substantially higher prediction performance. In our three-way data split, the testing set is used for the purpose of independent testing only and the validation set is used for tuning the parameters in the SVM training. Splitting the data three ways to get training, validation, and testing sets actually makes our approach very close to real applications. We cannot always select markers based on testing data because in most real applications the testing data are blind or unknown pending for prediction. The prediction performance of the testing set in a three-way data split can actually reflect the outcome in a real application. The best model selected from the training group may not produce the best prediction performance in the testing data due to the inconsistence between the training data and testing data. However, our results show that the selected top models will produce acceptable performance in the testing set, although not best performance.

Although some other researches achieved higher performance, for example, $82 \%$ by Sharma et al. [8] and $79.5 \%$ by Aarøe et al. [7], our prediction result outperforms theirs if we use training group only (precision $=82.86 \%$, accuracy $=83.33 \%$, sensitivity $=85.29 \%$, and specificity $=81.25 \%$,
Table 4) as they did. Our prediction performance which is more close to a real application is actually based on the testing set which is totally blind to the training group (precision $=72.41 \%$, accuracy $=68.75 \%$, sensitivity $=63.64 \%$, and specificity $=74.19 \%$, Table 4 ).

One limitation of the three-way data split is the sample size. If we split a small size sample into three ways, we would end up with so little data in each set that our analysis would lack any power. If we identify the inconsistence of prediction performance between the validation set and testing set, we can increase the size of training group (training set and validation set) and decrease the size of testing set by simply moving some samples in the testing set to the training group.

Since our approach enumerates all possible combinations of 5 out of $N$ markers, there is a limitation for the size of $N$ due to current computational capability. In our talon supercomputer, it would take about 1 hour to calculate all combinations of 5 out of 32 markers and about two weeks to finish the computation of picking 5 out of 100 markers. It is acceptable for us to set the maximum of $N$ to be 100 because in most cases the top 100 markers can be both specific and sensitive in understanding the treatment, diagnosis, and prognosis of cancer and can be limited by setting a reasonable $P$ value threshold.

An ANOVA statistical model is used for identifying differentially expressed genes between cancer and normal samples. For a simple two-group comparison, we would get the identical result if we were to compare the two groups using ANOVA, $t$-test, or SAM. However, ANOVA is a much more flexible and powerful technique that can be applied to much more complex research issues with multiple factors than the other two methods. For example, for the peripheral blood data, we should take into account two factors: (1) the fixed group effect (caused by the experimental conditions or treatments being evaluated) and (2) the random sample effect (random effects from either individual biological samples or sample preparations). In this case, ANOVA method is more efficient than multiple two-group studies analyzed via $t$-test or SAM, because with fewer observations we can gain more information.

In this work, we use the support vector machine (SVM) for classification, which is in general believed to outperform the other classification methods such as the logistic regression (LR) and the artificial neural networks (ANN) $[19,20]$, because the SVM prediction improves LR and ANN significantly along the specificity axis [21]. However, we understand that for special problems the ANN may still yield reasonable results and that the conclusion that SVM outperforms ANN is in general from a theoretical perspective and in particular for the considered case study [22]. Therefore, we strongly suggest that the tree-way data split method should be carried out for this kind of comparison before we reach any conclusions.

\section{Conclusions}

We developed an integrated computational approach that addressed a challenging multipanel biomarker development problem in the early detection of breast cancer in peripheral blood. The approach that we used combined simple statistical 
filtering of ANOVA with an optimization model of SVM. The approach automatically learned nonlinear relationships between features and outcomes to generate predictive models, which achieved AUC $=0.7879$ performance with a sensitivity of $63.64 \%$ and a specificity of $74.19 \%$ in the testing data set of 33 women with breast cancer and 31 healthy women controls. The SVM combined with the AUC optimization method is capable of identifying the optimal combination of multimarkers for performance comparable to that of conventional medical decision support systems. We believe that this computational approach works well with early detection of breast cancer in peripheral blood and can provide general guidance for future molecular medicine multimarker panel discovery applications in other diseases. In the future, we will follow up with biological experiments to validate these biomarkers with our collaborators.

\section{Conflict of Interests}

All authors declare that there is no conflict of interests.

\section{Authors' Contribution}

Renee Drabier conceived the initial work and designed the method. Fan Zhang generated the datasets, developed the statistics method, and performed the statistical analyses of the case studies. Youping Deng validated markers for early detection of breast cancer in peripheral blood. All authors are involved in the drafting and revisions of the paper.

\section{Acknowledgment}

This work was supported by the bioinformatics program in the University of North Texas Health Science Center.

\section{References}

[1] X. Hu, Y. Zhang, A. Zhang et al., "Comparative serum proteome analysis of human lymph node negative/positive invasive ductal carcinoma of the breast and benign breast disease controls via label-free semiquantitative shotgun technology," OMICS: A Journal of Integrative Biology, vol. 13, no. 4, pp. 291-300, 2009.

[2] B. A. Zeidan, R. I. Cutress, N. Murray et al., "Proteomic analysis of archival breast cancer serum," Cancer Genomics and Proteomics, vol. 6, no. 3, pp. 141-148, 2009.

[3] A. Lebrecht, D. Boehm, M. Schmidt, H. Koelbl, R. L. Schwirz, and F. H. Grus, "Diagnosis of breast cancer by tear proteomic pattern," Cancer Genomics and Proteomics, vol. 6, no. 3, pp. 177$182,2009$.

[4] M. Suzuki and D. Tarin, "Gene expression profiling of human lymph node metastases and matched primary breast carcinomas: clinical implications," Molecular Oncology, vol. 1, no. 2, pp. 172-180, 2007.

[5] K. Polyak, "Breast cancer: origins and evolution," The Journal of Clinical Investigation, vol. 117, no. 11, pp. 3155-3163, 2007.

[6] F. Zhang and J. Y. Chen, "Discovery of pathway biomarkers from coupled proteomics and systems biology methods," BMC Genomics, vol. 11, supplement 2, article S12, 2010.
[7] J. Aarøe, T. Lindahl, V. Dumeaux et al., "Gene expression profiling of peripheral blood cells for early detection of breast cancer," Breast Cancer Research, vol. 12, no. 1, article R7, 2010.

[8] P. Sharma, N. S. Sahni, R. Tibshirani et al., "Early detection of breast cancer based on gene-expression patterns in peripheral blood cells," Breast Cancer Research, vol. 7, no. 5, pp. R634-644, 2005.

[9] A. Vlahou, C. Laronga, L. Wilson et al., "A novel approach toward development of a rapid blood test for breast cancer," Clinical Breast Cancer, vol. 4, no. 3, pp. 203-209, 2003.

[10] V. N. Vapnik, "An overview of statistical learning theory," IEEE Transactions on Neural Networks, vol. 10, no. 5, pp. 988-999, 1999.

[11] H. X. Liu, R. S. Zhang, F. Luan et al., "Diagnosing breast cancer based on support vector machines," Journal of Chemical Information and Computer Sciences, vol. 43, no. 3, pp. 900-907, 2003.

[12] C. Henneges, D. Bullinger, R. Fux et al., "Prediction of breast cancer by profiling of urinary RNA metabolites using Support Vector Machine-based feature selection," BMC Cancer, vol. 9, article 104, 2009.

[13] J. J. Liu, G. Cutler, W. Li et al., "Multiclass cancer classification and biomarker discovery using GA-based algorithms," Bioinformatics, vol. 21, no. 11, pp. 2691-2697, 2005.

[14] F. Zhang and J. Y. Chen, "A Neural network approach to multibiomarker panel development based on LC/MS/MS proteomics profiles: a case study in breast cancer," in Proceedings of the 22nd IEEE International Symposium on Computer-Based Medical Systems (CBMS '09), pp. 1-6, August 2009.

[15] J. D. Storey and R. Tibshirani, "Statistical significance for genomewide studies," Proceedings of the National Academy of Sciences of the United States of America, vol. 100, pp. 9440-9445, 2003.

[16] D. Meyer, F. Leisch, and K. Hornik, "The support vector machine under test," Neurocomputing, vol. 55, no. 1-2, pp. 169186, 2003.

[17] F. Zhang and R. Drabier, "IPAD: the integrated pathway analysis database for systematic enrichment analysis," BMC Bioinformatics, vol. 13, supplement 15, article S7, 2012.

[18] W. Li, "How many genes are needed for early detection of breast cancer, based on gene expression patterns in peripheral blood cells?" Breast Cancer Research, vol. 7, no. 5, p. E5, 2005.

[19] N. Cristianini and J. Shawe-Taylor, An Introduction to Support Vector Machines, Cambridge University Press, Cambridge, Mass, USA, 2000.

[20] J. V. Tu, "Advantages and disadvantages of using artificial neural networks versus logistic regression for predicting medical outcomes," Journal of Clinical Epidemiology, vol. 49, no. 11, pp. 1225-1231, 1996.

[21] F. Dal Moro, A. Abate, G. R. G. Lanckriet et al., "A novel approach for accurate prediction of spontaneous passage of ureteral stones: support vector machines," Kidney International, vol. 69, no. 1, pp. 157-160, 2006.

[22] A. Abate, F. Dal Moro, and G. R. G. Lanckriet, "Response to 'support vector machines versus artificial neural network: who is the winner?"' Kidney International, vol. 71, no. 1, pp. 84-85, 2007. 

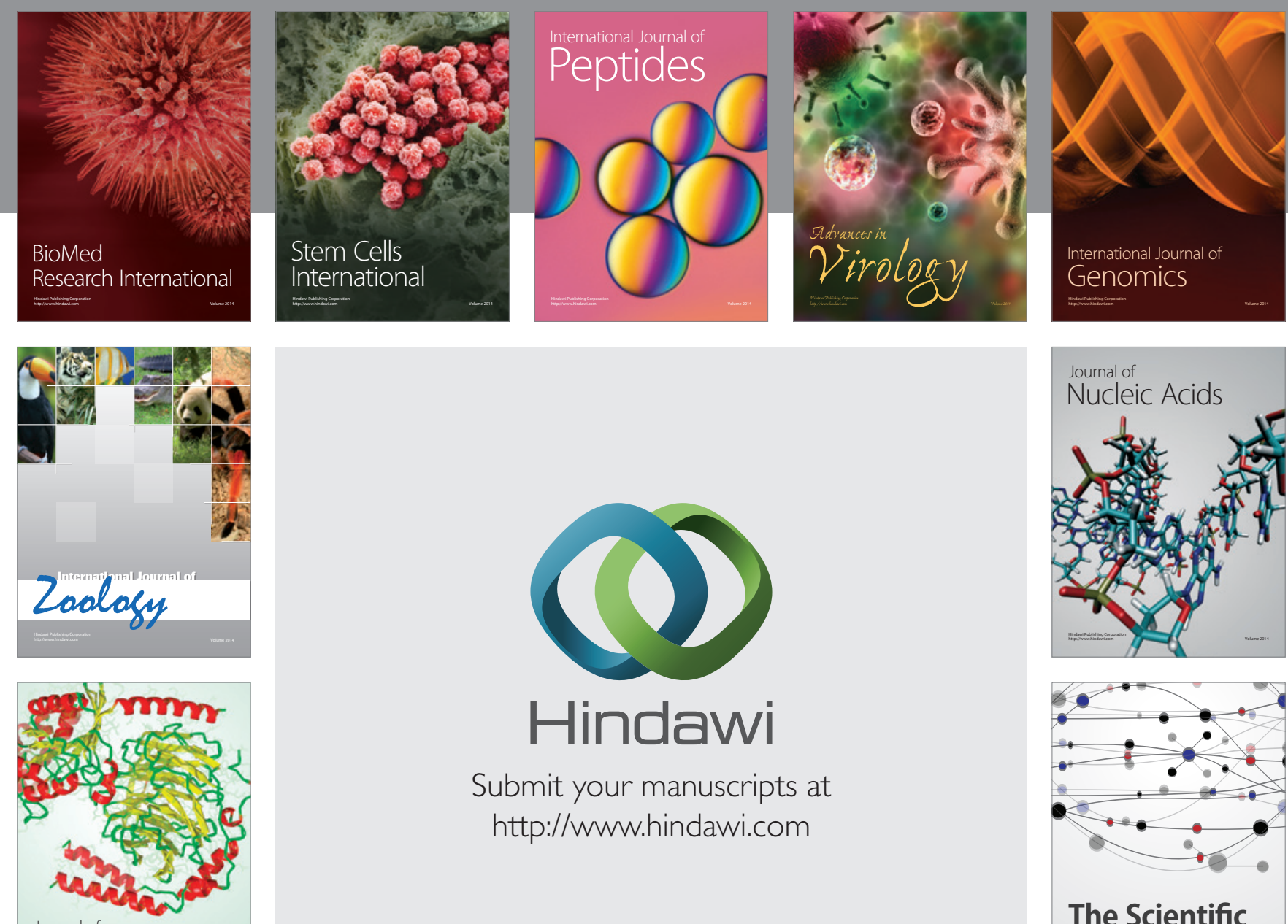

Submit your manuscripts at

http://www.hindawi.com

Journal of
Signal Transduction
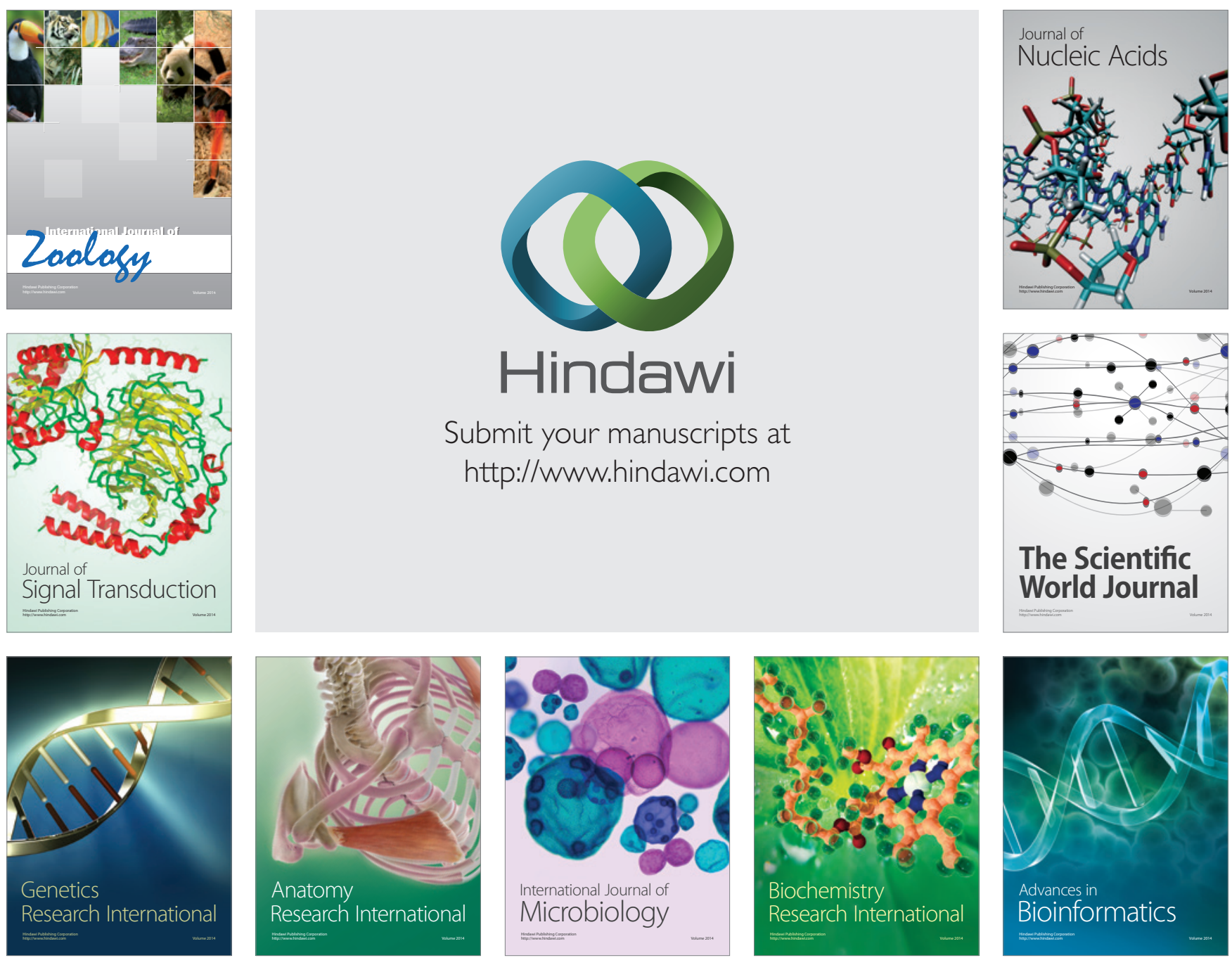

The Scientific World Journal
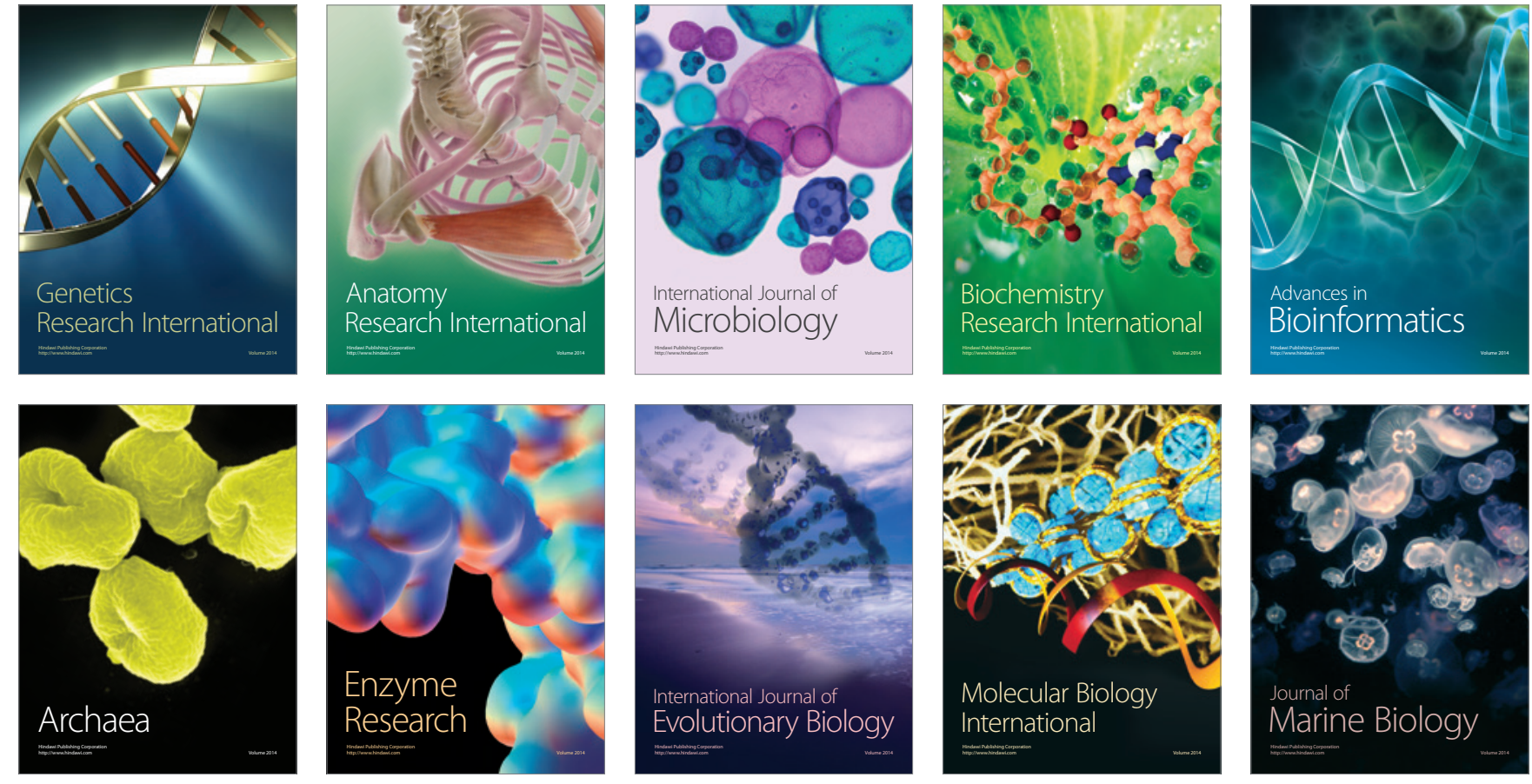